\title{
Factors associated with posttraumatic meningitis among traumatic head injury patients: a nationwide study in Japan
}

\author{
Yusuke Katayama ${ }^{1} \cdot$ Tetsuhisa Kitamura $^{2} \odot \cdot$ Kosuke Kiyohara $^{3}\left[\right.$ Junya Sado $^{2} \cdot$ Tomoya Hirose $^{1,4}$. \\ Tasuku Matsuyama $^{5}$ - Takeyuki Kiguchi ${ }^{6}$ (D) Jotaro Tachino ${ }^{1}$. Shunichiro Nakao ${ }^{1}$ - Yutaka Umemura ${ }^{1,7}$. \\ Yuko Nakagawa ${ }^{1} \cdot$ Takeshi Shimazu $^{1}$
}

Received: 29 June 2019 / Accepted: 28 August 2019 / Published online: 3 September 2019

(c) The Author(s) 2019

\begin{abstract}
Purpose Posttraumatic meningitis is one of the severe complications that can result in increased mortality and longer hospital stay among trauma patients. Factors such as cerebrospinal fluid (CSF) fistula and basilar skull fracture are associated with posttraumatic meningitis. However, it remains unclear whether procedures such as burr hole surgery in the emergency department and decompressive craniectomy are associated with posttraumatic meningitis. The aim of this study was to assess factors associated with posttraumatic meningitis with a nationwide hospital-based trauma registry in Japan.

Methods This was a retrospective observational study with a 12-year study period from January 2004 to December 2015. We included trauma patients registered in the Japanese Trauma Data Bank, whose head Abbreviated Injury Scale score was $\geq 3$ in this study. The main endpoint was the occurrence of meningitis during hospitalization. Multivariable logistic regression analysis was used to assess independent parameters associated with posttraumatic meningitis such as CSF fistula, burr hole surgery in the emergency department, and decompressive craniectomy.

Results Among 60,390 head injury patients with head AIS score 3 or more, $284(0.5 \%)$ patients had posttraumatic meningitis. Factors associated with posttraumatic meningitis were burr hole surgery in the emergency department (adjusted odds ratio [AOR] 2.158 [95\% confidence interval (CI) 1.401-3.325]), decompressive craniectomy (AOR 2.123 [95\% CI 1.506-2.993]), external ventricular drainage (AOR 1.843 [95\% CI, 1.157-2.935]), CSF leakage (AOR 3.328 [95\% CI 2.205-5.022]), and basilar skull fracture (AOR 1.651 [95\% CI 1.178-2.314]).
\end{abstract}

Conclusions In this population of trauma patients, burr hole surgery in the emergency department and decompressive craniectomy was associated with posttraumatic meningitis.

Keywords Posttraumatic meningitis · Decompressive craniectomy $\cdot$ Cerebrospinal fluid leakage $\cdot$ Burr hole surgery in emergency department

Yusuke Katayama

orion13@hp-emerg.med.osaka-u.ac.jp

1 Department of Traumatology and Acute Critical Medicine, Osaka University Graduate School of Medicine, 2-15, Yamada-oka, Suita, Japan

2 Division of Environmental Medicine and Population Sciences, Department of Social and Environmental Medicine, Osaka University Graduate School of Medicine, 2-15, Yamada-oka, Suita, Japan

3 Department of Food Science, Faculty of Home Economics, Otsuma Women's University Tokyo, 12, Sanban-cho,

Chiyoda-ku, Tokyo, Japan
4 Emergency and Critical Care Center, Osaka Police Hospital, 10-31, Kitayama-cho, Tennoji-ku, Osaka, Japan

5 Department of Emergency Medicine, Kyoto Prefectural University of Medicine, 465 Kajiicho, Hiroko-ji noboru, Kawaramachi-dori, Kamigyo-ku, Kyoto, Japan

6 Kyoto University Health Services, Yoshida-honmachi, Sakyo-ku, Kyoto, Japan

7 Department of Emergency and Critical Care, Osaka General Medical Center, 3-1-56, Bandai-Higashi, Sumiyoshi-ku, Osaka, Japan 


\section{Introduction}

Head injury occurs in about $25 \%$ of trauma patients in Japan [1], and mortality remains high. In addition, many head injury survivors have a poor neurological outcome due to diffuse axonal injury [2]. Meningitis can be a severe complication with a negative influence on the outcome after craniocerebral trauma. There are reports about high mortality rates from $29 \%$ to $57.9 \%$ due to this infection [3-5]. Previous studies revealed that basilar skull fracture and cerebrospinal fluid (CSF) leakage were also associated with posttraumatic meningitis $[6,7]$. In addition, the length of time of external ventricular drainage, emergency operation, and operation time over $4.5 \mathrm{~h}$ was also reported to relate to the occurrence of postoperative meningitis in patients undergoing a neurosurgical operation $[8,9]$.

If it takes time to transport severe trauma patients from areas, where there are no neurosurgeons to distant medical institutions, their prognosis would become worse [10]. In addition, it may take time to prepare a hospital operating room when an emergency operation is required for severe head injury patients. Thus, burr hole surgery is sometimes performed in the emergency department for some patients, and decompressive craniectomy for patients with traumatic brain injury and refractory intracranial hypertension has resulted in a favorable neurological outcome [11-15]. However, it is unclear whether burr hole surgery performed in the emergency department, the procedures performed during the neurosurgical operation and repeat surgery are associated with posttraumatic meningitis in patients with traumatic head injury.

The Japanese Trauma Data Bank (JTDB) is a nationwide trauma registry in Japan that is managed by The Japanese Association for The Surgery of Trauma. Data registration in the JTDB was launched in 2003, and approximately 230,000 emergency trauma patients were enrolled by 2015 [1]. With the use of data from the JTDB registry, the aim of this study was to evaluate the association between the occurrence of posttraumatic meningitis and factors such as patient characteristics, type of surgery, and procedures performed during the surgery among the traumatic head injury patients with head AIS score 3 or more.

\section{Methods}

\section{Study design, population, and setting}

This was a retrospective observational study that used data of emergency trauma patients registered in the JTDB. The study period was the 12-year period from January 2004 to December 2015. In this study, we included patients with a head Abbreviated Injury Scale (AIS) score of 3 or more who were transported to a JTDB-participating hospital and were registered in the JTDB registry [16]. We excluded those patients who were in cardiopulmonary arrest on hospital arrival, whose mandatory data were missing. Cardiopulmonary arrest on hospital arrival was defined as a patient, whose systolic blood pressure was $0 \mathrm{mmHg}$ and/or heart rate was $0 \mathrm{bpm}$ on hospital arrival. From the JTDB database, we extracted factors such as age, sex, past medical history, type of trauma, CSF leakage, type of skull fracture, burr hole surgery in the emergency department, operative procedure, and procedures performed during the neurosurgery, and repeat surgery within $48 \mathrm{~h}$ of the first surgery. In this study, we defined multiple trauma as head trauma patients with AIS score of 3 or more in body areas other than the head. In addition, we defined CSF leakage as a patient for whom AIS code 150204.3 was recorded. In addition, those with AIS codes 150408.4, 150406.4, $150404.3,150402.2$, and 150400.2 were defined as having a skull fracture, and those with AIS codes 150206.4, 150204.3, 150202.3, and 150200.3 were defined as having a basilar skull fracture. The primary operative method of neurosurgery was classified as burr hole surgery or craniotomy, and the procedures performed during the neurosurgery were classified as evacuation of hematoma, decompressive craniectomy, lobectomy, duraplasty, cranioplasty, and external ventricular drainage. This study was approved by the ethics committee of Osaka University Graduate School of Medicine (No. 16260). Personnel identifiers were removed beforehand from the JTDB database, and thus, the patients' right to informed consent was waived.

\section{Japanese Trauma Data Bank}

The JTDB was launched in 2003 by the Japanese Association for the Surgery of Trauma (Trauma Surgery Committee) and the Japanese Association for Acute Medicine (Committee for Clinical Care Evaluation) [1, 17], similar to trauma databases in North America, Europe, and Oceania [18]. By 2016, 256 major emergency medical institutions across Japan had been registered in the JTDB database [1]. These hospitals have equal ability to that of Level I trauma centers in the United States. Data were collected via the Internet from participating institutions. The physicians and medical assistants who attended the AIS-coding course were the main registers of the data [19].

The JTDB captures trauma patient data on age, sex, mechanism of injury, AIS code (version 1998), Injury Severity Score (ISS), vital signs on hospital arrival, date and time series from hospital arrival to discharge, medical treatments such as interventional radiology, surgical operation, and CT 
scanning, complications, and mortality at discharge [19]. ISS was calculated from the top three scores of AIS in nine sites classified by AIS codes.

\section{Endpoint}

The endpoint was the occurrence of meningitis during hospitalization, and we defined this meningitis as posttraumatic meningitis. We extracted data on the occurrence of meningitis from the JTDB registry.

\section{Statistical analysis}

In this study, we assessed factors associated with the occurrence of posttraumatic meningitis with multivariable logistic regression analysis and calculated the adjusted odds ratio (AOR) and 95\% confidential interval (CI). Multivariable logistic regression analysis was performed by forced enter-method. The independent parameters were age group (0-9 years, $10-19$ years, $20-29$ years, $30-39$ years, 40-49 years, 50-59 years, 60-69 years, 70-79 years, 80 years, or older), sex, diabetes, implementation of hemodialysis, multiple trauma or single head trauma, presence or absence of CSF leakage, type of skull fracture, implementation of burr hole surgery in the emergency department, first neurosurgical operation, procedures performed during the first neurosurgical operation, and repeat surgery within $48 \mathrm{~h}$ of the first surgery. These independent parameters that were biologically essential and considered to be associated with outcomes were included in the multivariable regression analysis-based previous studies [3-9, 20, 21]. As a further sub-analysis, we divided these patients into the single head trauma group and the multiple trauma group [22], and these independent parameters associated with posttraumatic meningitis were also assessed with multivariable logistic regression analysis. All tests were two-tailed, and a $P$ value of $<0.05$ was considered statistically significant. Statistical analysis was performed by SPSS version 23.0J (IBM Crop., Armonk, NY, USA). This manuscript was written based on the STROBE statement to assess the reporting of cohort and cross-sectional studies.

\section{Results}

Figure 1 shows the patient flow in this study. In total, 226,698 emergency patients were registered in the JTDB from 2004 to 2015 , and 66,818 patients had head trauma with a head AIS score of 3 or more. Among these head trauma patients, 4901 patients were in cardiopulmonary arrest on hospital arrival, and 1527 patients who did not have the necessary data (missing age: 65 patients, missing sex: 16 patients, and missing outcome data: 1446 patients)

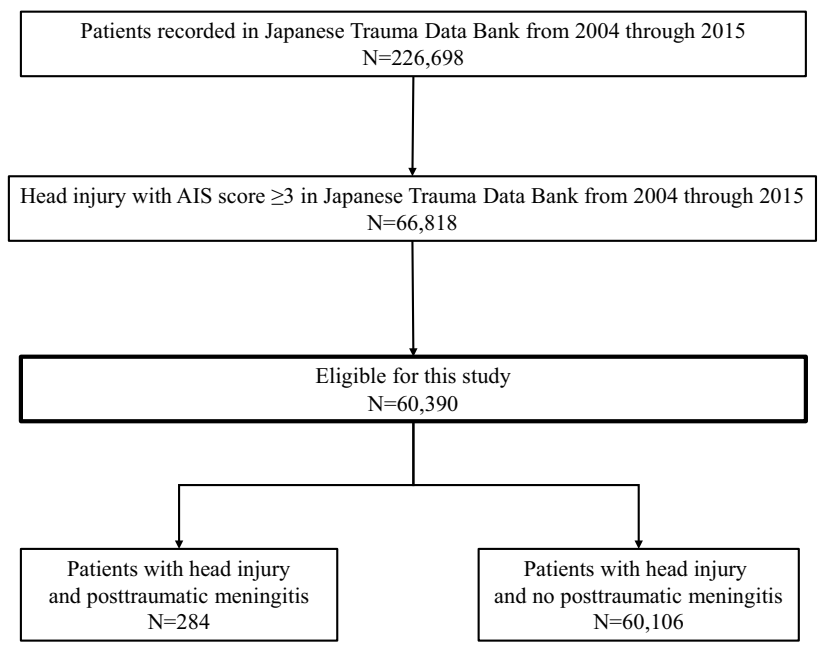

Fig. 1 Patient flow in this study

were excluded from this study, leaving 60,390 patients suitable for analysis. Among these 60,390 patients, 284 (0.5\%) had posttraumatic meningitis.

Table 1 shows the patient characteristics in this study. The median age was 63 (interquartile range [IQR] 38-76) years, $68.4 \%$ were males, and the median Glasgow Coma Scale score was 13 (IQR 8-15). In total, 14,085 patients (23.3\%) had skull fractures and 8388 patients $(13.9 \%)$ had basilar skull fractures. There were 2033 patients (3.4\%) with CSF leakage, and 1468 patients (2.4\%) had undergone burr hole surgery in the emergency department. As the first neurosurgical operation, craniotomy was performed in 7128 patients $(11.5 \%)$, and burr hole surgery was performed in $2910(4.5 \%)$. The procedures performed during the first neurosurgical operation included evacuation of hematoma in 8262 patients (13.7\%), decompressive craniectomy in $2495(4.1 \%)$, lobectomy in $416(0.7 \%)$, duraplasty in 267 $(0.4 \%)$, cranioplasty in $437(0.7 \%)$, and external ventricular drainage in 786 patients (1.3\%). Among those patients who underwent repeat surgery within $48 \mathrm{~h}$ of the first surgery, $928(1.5 \%)$ received a craniotomy and $235(0.4 \%)$ burr hole surgery.

Table 2 shows the results of the association between the occurrence of posttraumatic meningitis and various factors. Male sex (AOR 1.472 [95\% CI 1.102-1.965]), multiple trauma (AOR 1.415 [95\% CI 1.054-1.900]), basilar skull fracture (AOR 1.651 [95\% CI 1.178-2.314]), CSF leakage (AOR 3.328 [95\% CI 2.205-5.022]), burr hole surgery in the emergency department (AOR 2.158 [95\% CI 1.401-3.325]), craniotomy (AOR 4.629 [95\% CI 3.087-6.942]), and burr hole surgery as an operative method (AOR 2.259 [95\% CI 1.449-3.523]) were associated with the occurrence of posttraumatic meningitis. Regarding the procedure performed during the first neurosurgical operation, decompressive 
Table 1 Demographic and clinical characteristics of head injury patients with head AIS $\geq 3$

\begin{tabular}{|c|c|}
\hline Characteristic & Total $(n=60,390)$ \\
\hline Age, years, median (IQR) & $63(38-76)$ \\
\hline $0-9$ & $2574(4.3)$ \\
\hline $10-19$ & $4306(7.1)$ \\
\hline $20-29$ & $4695(7.8)$ \\
\hline $30-39$ & $4196(6.9)$ \\
\hline $40-49$ & $4818(8.0)$ \\
\hline $50-59$ & $6285(10.4)$ \\
\hline $60-69$ & $10,394(17.2)$ \\
\hline $70-79$ & $12,181(20.1)$ \\
\hline Over 80 & $10,941(18.1)$ \\
\hline Male, $n(\%)$ & $41,334(68.4)$ \\
\hline Glasgow Coma Scale, median (IQR) & $13(8-15)$ \\
\hline \multicolumn{2}{|l|}{ Past medical history, $n(\%)$} \\
\hline Diabetes mellitus & $5672(9.4)$ \\
\hline Dialysis & $861(1.4)$ \\
\hline \multicolumn{2}{|l|}{ Type of trauma, $n(\%)$} \\
\hline Single TBI & $50,975(84.4)$ \\
\hline Multiple trauma & 9415 (15.6) \\
\hline \multicolumn{2}{|l|}{ Type of skull fracture, $n(\%)$} \\
\hline Skull fracture & $14,085(23.3)$ \\
\hline Basilar skull fracture & $8388(13.9)$ \\
\hline Cerebrospinal fluid leakage, $n(\%)$ & $2033(3.4)$ \\
\hline Burr hole surgery in the emergency department, $n(\%)$ & $1468(2.4)$ \\
\hline \multicolumn{2}{|l|}{ First neurosurgical operation, $n(\%)$} \\
\hline Craniotomy & $7128(11.8)$ \\
\hline Burr hole surgery & $2910(4.8)$ \\
\hline \multicolumn{2}{|c|}{ Procedures performed during the first neurosurgical operation, $n(\%)$} \\
\hline Evacuation of hematoma & $8262(13.7)$ \\
\hline Decompressive craniectomy & $2495(4.1)$ \\
\hline Lobectomy & $416(0.7)$ \\
\hline Duraplasty & $267(0.4)$ \\
\hline Cranioplasty & $437(0.7)$ \\
\hline External ventricular drainage & $786(1.3)$ \\
\hline \multicolumn{2}{|c|}{ Repeat surgical procedure performed within $48 \mathrm{~h}$ of first surgery, $n(\%)$} \\
\hline Craniotomy & $928(1.5)$ \\
\hline Burr hole surgery & $235(0.4)$ \\
\hline
\end{tabular}

AIS Abbreviated Injury Scale, IQR interquartile range, $T B I$ traumatic brain injury craniectomy (AOR 2.123 [95\% CI 1.506-2.993]) and external ventricular drainage (AOR 1.843 [95\% CI 1.157-2.935]) were associated with posttraumatic meningitis. For repeat surgery within $48 \mathrm{~h}$ of the first surgery, both craniotomy (AOR; 3.193 [95\% CI 2.137-4.769]) and burr hole surgery (AOR; 4.222 [95\% CI 2.194-8.124]) were associated with posttraumatic meningitis.

Table 3 shows the results of sub-analysis divided into single head trauma and multiple trauma. In the group with single head trauma, burr hole surgery in the emergency department (AOR 2.456 [95\% CI 1.522-3.965]), craniotomy (AOR 3.587 [95\% CI 2.248-5.723]), and burr hole surgery as an operative method (AOR 2.112 [95\% CI 1.277-3.493]) were associated with posttraumatic meningitis. As a procedure performed during the first neurosurgical operation, only decompressive craniectomy (AOR 2.292 [95\% CI 1.562-3.363]) was associated with posttraumatic meningitis. In addition, both craniotomy (AOR 3.044 [95\% CI 1.952-4.746]) and burr hole surgery (AOR 3.387 [95\% CI 1.531-7.492]) were associated with posttraumatic meningitis when repeat surgery was performed within $48 \mathrm{~h}$ of the first surgery. In contrast, only craniotomy as a neurosurgical operative method (AOR 9.381 [95\% CI 4.199-20.958]) was associated with posttraumatic 
Table 2 Factors associated with posttraumatic meningitis

\begin{tabular}{lll}
\hline $\begin{array}{l}\text { Meningitis } \\
\%(n / N)\end{array}$ & Adjusted OR $(95 \% \mathrm{CI})$ & $P$ value \\
\hline
\end{tabular}

\section{Age group (years)}

$\begin{array}{llll}0-9 & 0.5(12 / 2574) & 0.923(0.461-1.847) & 0.821 \\ 10-19 & 0.5(20 / 4306) & 0.718(0.398-1.292) & 0.269 \\ 20-29 & 0.8(37 / 4695) & 1.239(0.750-2.049) & 0.403 \\ 30-39 & 0.7(29 / 4196) & \text { Reference } & \\ 40-49 & 0.5(26 / 4818) & 0.849(0.493-1.464) & 0.557 \\ 50-59 & 0.6(38 / 6285) & 1.041(0.631-1.716) & 0.876 \\ 60-69 & 0.5(47 / 10,394) & 0.765(0.473-1.239) & 0.277 \\ 70-79 & 0.4(46 / 12,181) & 0.797(0.489-1.297) & 0.360 \\ \text { Over 80 } & 0.3(29 / 10,941) & 0.749(0.436-1.286) & 0.294 \\ \text { Sex } & & & \\ \text { Male } & 0.5(221 / 41,334) & 1.472(1.102-1.965) & 0.009 \\ \text { Female } & 0.3(63 / 19,056) & \text { Reference } & \end{array}$

$0.3(63 / 19,056) \quad$ Reference

Past medical history

Diabetes mellitus

$\begin{array}{cllc}(+) & 0.4(25 / 5672) & 1.134(0.735-1.750) & 0.571 \\ (-) & 0.5(259 / 54,718) & \text { Reference } & \\ \begin{array}{c}\text { Dialysis } \\ (+)\end{array} & 0.3(3 / 861) & 0.925(0.290-2.959) & 0.896 \\ (-) & 0.5(281 / 59,529) & \text { Reference } & \end{array}$

Type of trauma

$\begin{array}{cll}\begin{array}{c}\text { Single } \\ \text { TBI }\end{array} & 0.4(223 / 50,975) & \text { Reference } \\ \begin{array}{c}\text { Multiple } \\ \text { trauma }\end{array} & 0.6(61 / 9415) & 1.415(1.054-1.900)\end{array}$

Type of skull fracture

Skull fracture
$(+)$
$0.8(108 / 14,085)$
$1.163(0.900-1.504)$
$(-)$
$0.4(176 / 46,305)$
Reference

Basilar skull fracture

$\begin{array}{clll}(+) & 1.2(99 / 8388) & 1.651(1.178-2.314) & 0.004 \\ (-) & 0.4(185 / 52,002) & \text { Reference } & \\ \text { CSF leakage } & & & \\ (+) & 2.7(54 / 2033) & 3.328(2.205-5.022) & <0.001 \\ (-) & 0.4(230 / 58,127) & \text { Reference } & \end{array}$

Burr hole surgery in ED

$\begin{array}{llll}(+) & 3.3(49 / 1468) & 2.158(1.401-3.325) & <0.001 \\ (-) & 0.4(235 / 58,922) & \text { Reference }\end{array}$

First neurosurgical operation

Craniotomy

$$
\begin{array}{llll}
(+) & 1.8(125 / 7128) & 4.629(3.087-6.942) & <0.001 \\
(-) & 0.3(159 / 53,262) & \text { Reference }
\end{array}
$$

Burr hole surgery

$$
\begin{array}{llll}
(+) & 1.9(54 / 2910) & 2.259(1.449-3.523) & <0.001 \\
(-) & 0.4(230 / 57,480) & \text { Reference }
\end{array}
$$

Procedures performed during the first neurosurgical operation

\begin{tabular}{|c|c|c|c|}
\hline & $\begin{array}{l}\text { Meningitis } \\
\%(n / N)\end{array}$ & Adjusted OR (95\% CI) & $P$ value \\
\hline \multicolumn{4}{|c|}{ Decompressive craniectomy } \\
\hline$(+)$ & $3.4(84 / 2495)$ & $2.123(1.506-2.993)$ & $<0.001$ \\
\hline$(-)$ & $0.3(200 / 57,895)$ & Reference & \\
\hline \multicolumn{4}{|c|}{ Lobectomy } \\
\hline$(+)$ & $2.9(12 / 416)$ & $0.871(0.460-1.651)$ & 0.673 \\
\hline$(-)$ & $0.5(272 / 59,974)$ & Reference & \\
\hline \multicolumn{4}{|c|}{ Duraplasty } \\
\hline$(+)$ & $2.6(7 / 267)$ & $0.871(0.384-1.975)$ & 0.740 \\
\hline$(-)$ & $0.5(477 / 60,123)$ & Reference & \\
\hline \multicolumn{4}{|c|}{ Cranioplasty } \\
\hline$(+)$ & $2.1(9 / 437)$ & $1.284(0.619-2.663)$ & 0.502 \\
\hline$(-)$ & $0.5(275 / 59,953)$ & Reference & \\
\hline \multicolumn{4}{|c|}{ External ventricular drainage } \\
\hline$(+)$ & $3.6(28 / 786)$ & $1.843(1.157-2.935)$ & 0.010 \\
\hline$(-)$ & $0.4(256 / 59,604)$ & Reference & \\
\hline \multicolumn{4}{|c|}{ Repeat surgical procedure performed within $48 \mathrm{~h}$ of first surgery } \\
\hline \multicolumn{4}{|c|}{ Craniotomy } \\
\hline$(+)$ & $4.6(43 / 928)$ & $3.193(2.137-4.769)$ & $<0.001$ \\
\hline$(-)$ & $0.4(241 / 59,462)$ & Reference & \\
\hline \multicolumn{4}{|c|}{ Burr hole surgery } \\
\hline$(+)$ & $5.1(12 / 235)$ & $4.222(2.194-8.124)$ & $<0.001$ \\
\hline$(-)$ & $0.5(272 / 60,155)$ & Reference & \\
\hline
\end{tabular}
Evacuation of hematoma

\begin{tabular}{llll}
$(+)$ & $1.6(134 / 8262)$ & $0.792(0.528-1.188)$ & 0.260 \\
$(-)$ & $0.3(150 / 52,128)$ & Reference & \\
\hline
\end{tabular}

Table 2 (continued)

$T B I$ traumatic brain injury, $C S F$ cerebrospinal fluid, $E D$ emergency department, $O R$ odds ratio, $C I$ confidence interval

meningitis in the multiple trauma group. As a procedure performed during the first neurosurgical operation, external ventricular drainage (AOR 2.790 [95\% CI 1.113-6.992]) was associated with posttraumatic meningitis, but the evacuation of hematoma was inversely related (AOR 0.417 [95\% CI 0.179-0.970]). In addition, both craniotomy (AOR 4.372 [95\% CI 1.661-11.503]) and burr hole surgery (AOR 6.368 [95\% CI 1.825-22.224]) were associated with posttraumatic meningitis as a repeat surgical procedure performed within $48 \mathrm{~h}$ of the first surgery.

\section{Discussion}

Using data from the JTDB as a nationwide hospital-based trauma registry in Japan, this study revealed that factors such as male sex, multiple trauma, basilar skull fracture, CSF leakage, burr hole surgery in the emergency department, burr hole surgery and craniotomy as operative methods for the first surgery, decompressive craniectomy and external ventricular drainage as procedures performed during the first neurosurgical operation, and repeat surgery within $48 \mathrm{~h}$ of the first surgery were associated with posttraumatic 
Table 3 Odds ratios of each variable for posttraumatic meningitis among patients with single TBI and multiple trauma

\begin{tabular}{|c|c|c|c|c|c|c|}
\hline & \multicolumn{3}{|l|}{ Single TBI } & \multicolumn{3}{|c|}{ Multiple trauma } \\
\hline & $\%(n / N)$ & Adjusted OR (95\% CI) & $P$ value & $\%(n / N)$ & Adjusted OR (95\% CI) & $P$ value \\
\hline \multicolumn{7}{|c|}{ Age group (years) } \\
\hline $0-9$ & $0.3(8 / 2325)$ & $0.662(0.293-1.496)$ & 0.321 & $1.6(4 / 249)$ & $3.188(0.736-13.805)$ & 0.121 \\
\hline $10-19$ & $0.3(11 / 3591)$ & $0.461(0.223-0.953)$ & 0.037 & $1.3(9 / 715)$ & $2.409(0.711-8.164)$ & 0.158 \\
\hline 20-29 & $0.8(28 / 3700)$ & $1.107(0.633-1.938)$ & 0.721 & $0.9(9 / 995)$ & $2.166(0.647-7.247)$ & 0.210 \\
\hline $30-39$ & $0.8(25 / 3319)$ & Reference & & $0.5(4 / 877)$ & Reference & \\
\hline $40-49$ & $0.4(17 / 3952)$ & $0.632(0.336-1.190)$ & 0.155 & $1.0(9 / 866)$ & $2.403(0.715-8.083)$ & 0.156 \\
\hline $50-59$ & $0.6(33 / 5237)$ & $0.972(0.567-1.668)$ & 0.918 & $0.5(5 / 1048)$ & $1.291(0.336-4.959)$ & 0.710 \\
\hline $60-69$ & $0.4(37 / 8838)$ & $0.653(0.384-1.110)$ & 0.115 & $0.6(10 / 1556)$ & $1.425(0.431-4.709)$ & 0.562 \\
\hline 70-79 & $0.4(40 / 10,451)$ & $0.749(0.442-1.270)$ & 0.284 & $0.3(6 / 1730)$ & $0.960(0.261-3.538)$ & 0.952 \\
\hline over 80 & $0.3(24 / 9562)$ & $0.659(0.364-1.191)$ & 0.167 & $0.4(5 / 1379)$ & $1.391(0.358-5.414)$ & 0.634 \\
\hline \multicolumn{7}{|l|}{ Sex } \\
\hline Male & $0.5(173 / 34,904)$ & $1.425(1.029-1.974)$ & 0.033 & $0.7(48 / 6430)$ & $1.533(0.812-2.891)$ & 0.187 \\
\hline Female & $0.3(50 / 16,071)$ & Reference & & $0.4(13 / 2985)$ & Reference & \\
\hline \multicolumn{7}{|c|}{ Past medical history } \\
\hline \multicolumn{7}{|c|}{ Diabetes mellitus } \\
\hline$(+)$ & $0.5(23 / 5010)$ & $1.225(0.775-1.935)$ & 0.386 & $0.3(2 / 662)$ & $0.659(0.155-2.815)$ & 0.574 \\
\hline$(-)$ & $0.4(200 / 45,965)$ & Reference & & $0.7(59 / 8753)$ & Reference & \\
\hline \multicolumn{7}{|l|}{ Dialysis } \\
\hline$(+)$ & $0.3(2 / 793)$ & $0.666(0.161-2.747)$ & 0.574 & $1.5(1 / 68)$ & $3.771(0.468-30.402)$ & 0.213 \\
\hline$(-)$ & $0.4(221 / 50,182)$ & Reference & & $0.6(60 / 9347)$ & Reference & \\
\hline \multicolumn{7}{|c|}{ Type of fracture } \\
\hline \multicolumn{7}{|c|}{ Skull fracture } \\
\hline$(+)$ & $0.7(90 / 12,102)$ & $1.218(0.913-1.624)$ & 0.179 & $0.9(18 / 1983)$ & $1.023(0.564-1.856)$ & 0.941 \\
\hline$(-)$ & $0.3(133 / 38,873)$ & Reference & & $0.6(43 / 7432)$ & Reference & \\
\hline \multicolumn{7}{|c|}{ Skull base fracture } \\
\hline$(+)$ & $1.2(81 / 6853)$ & $1.830(1.251-2.676)$ & 0.002 & $1.2(18 / 1535)$ & $1.253(0.595-2.640)$ & 0.553 \\
\hline$(-)$ & $0.3(142 / 44,122)$ & Reference & & $0.5(43 / 7880)$ & Reference & \\
\hline \multicolumn{7}{|c|}{ CSF leakage } \\
\hline$(+)$ & $2.8(45 / 1623)$ & $3.543(2.244-5.723)$ & $<0.001$ & $2.2(9 / 410)$ & $2.334(0.874-6.235)$ & 0.091 \\
\hline$(-)$ & $0.4(178 / 49,352)$ & Reference & & $0.6(52 / 9005)$ & Reference & \\
\hline \multicolumn{7}{|c|}{ Burr hole surgery at ED } \\
\hline$(+)$ & $3.5(39 / 1118)$ & $2.456(1.522-3.965)$ & $<0.001$ & $2.9(10 / 350)$ & $1.620(0.611-4.291)$ & 0.332 \\
\hline$(-)$ & $0.4(184 / 49,857)$ & Reference & & $0.651 / 9065$ & Reference & \\
\hline \multicolumn{7}{|c|}{ First neurosurgical operation } \\
\hline \multicolumn{7}{|c|}{ Craniotomy } \\
\hline$(+)$ & $1.6(101 / 6271)$ & $3.587(2.248-5.723)$ & $<0.001$ & $2.8(24 / 857)$ & $9.381(4.199-20.958)$ & $<0.001$ \\
\hline$(-)$ & $0.3(110 / 43,692)$ & Reference & & $0.4(37 / 8558)$ & Reference & \\
\hline \multicolumn{7}{|c|}{ Burr hole surgery } \\
\hline$(+)$ & $1.7(43 / 2471)$ & $2.112(1.277-3.493)$ & 0.004 & $2.5(11 / 439)$ & $2.278(0.873-5.944)$ & 0.092 \\
\hline$(-)$ & $0.4(180 / 48,504)$ & Reference & & $0.6(50 / 8976)$ & Reference & \\
\hline \multicolumn{7}{|c|}{ Procedures performed during the first neurosurgical operation } \\
\hline \multicolumn{7}{|c|}{ Evacuation of hematoma } \\
\hline$(+)$ & $1.6(113 / 7283)$ & $0.988(0.618-1.580)$ & 0.988 & $2.1(21 / 979)$ & $0.417(0.179-0.970)$ & 0.042 \\
\hline$(-)$ & $0.3(110 / 43,692)$ & Reference & & $0.5(40 / 8436)$ & Reference & \\
\hline \multicolumn{7}{|c|}{ Decompressive craniectomy } \\
\hline$(+)$ & $3.3(69 / 2120)$ & $2.292(1.562-3.363)$ & $<0.001$ & $4.0(15 / 375)$ & $1.468(0.671-3.211)$ & 0.337 \\
\hline$(-)$ & $0.3(154 / 48,855)$ & Reference & & $0.5(46 / 9040)$ & Reference & \\
\hline
\end{tabular}


Table 3 (continued)

\begin{tabular}{|c|c|c|c|c|c|c|}
\hline & \multicolumn{3}{|l|}{ Single TBI } & \multicolumn{3}{|c|}{ Multiple trauma } \\
\hline & $\%(n / N)$ & Adjusted OR (95\% CI) & $P$ value & $\%(n / N)$ & Adjusted OR (95\% CI) & $P$ value \\
\hline \multicolumn{7}{|c|}{ Lobectomy } \\
\hline$(+)$ & $3.1(11 / 354)$ & $0.909(0.459-1.799)$ & 0.785 & $1.6(1 / 62)$ & $0.547(0.070-4.292)$ & 0.566 \\
\hline$(-)$ & $0.4(212 / 50,621)$ & Reference & & $0.660 / 9353$ & Reference & \\
\hline \multicolumn{7}{|c|}{ Duraplasty } \\
\hline$(+)$ & $3.2(7 / 222)$ & $1.165(0.510-2.662)$ & 0.717 & $0.0(0 / 45)$ & NA & - \\
\hline$(-)$ & $0.4(216 / 50,753)$ & Reference & & $0.7(61 / 9370)$ & Reference & \\
\hline \multicolumn{7}{|c|}{ Cranioplasty } \\
\hline$(+)$ & $2.1(8 / 382)$ & $1.522(0.697-3.324)$ & 0.292 & $1.8(1 / 55)$ & $0.877(0.111-6.933)$ & 0.901 \\
\hline$(-)$ & $0.4(215 / 50,356)$ & Reference & & $0.6(60 / 9360)$ & Reference & \\
\hline \multicolumn{7}{|c|}{ External ventricular drainage } \\
\hline$(+)$ & $3.1(19 / 619)$ & $1.584(0.915-2.740)$ & 0.100 & $5.4(9 / 167)$ & $2.790(1.113-6.992)$ & 0.029 \\
\hline$(-)$ & $0.4(204 / 50,356)$ & Reference & & $0.6(52 / 9248)$ & Reference & \\
\hline \multicolumn{7}{|c|}{ Repeat surgical procedure performed within $48 \mathrm{~h}$ of first surgery } \\
\hline \multicolumn{7}{|c|}{ Craniotomy } \\
\hline$(+)$ & $4.6(36 / 776)$ & $3.044(1.952-4.746)$ & $<0.001$ & $4.6(7 / 152)$ & $4.372(1.661-11.503)$ & 0.003 \\
\hline$(-)$ & $0.4(187 / 50,199)$ & Reference & & $0.6(54 / 9263)$ & Reference & \\
\hline \multicolumn{7}{|c|}{ Burr hole surgery } \\
\hline$(+)$ & $4.4(8 / 180)$ & $3.387(1.531-7.492)$ & 0.003 & $7.3(4 / 55)$ & $6.368(1.825-22.224)$ & 0.004 \\
\hline$(-)$ & $0.4(215 / 50,795)$ & Reference & & $0.6(57 / 9360)$ & Reference & \\
\hline
\end{tabular}

$n / N$ number of patients with posttraumatic meningitis/number of patients, $T B I$ traumatic brain injury, $E D$ emergency department, $O R$ odds ratio, CI confidence interval, NA not applicable

*When assessing one variable, we adjusted other variables listed in this table

meningitis among patients with traumatic brain injury patients with head AIS score 3 or more. In the subgroup analysis, burr hole surgery in the emergency department, craniotomy, decompressive craniectomy, and repeat surgery within $48 \mathrm{~h}$ were associated with posttraumatic meningitis in patients with single head trauma. In contrast, in multiple trauma patients, craniotomy, external ventricular drainage, and repeat surgery within $48 \mathrm{~h}$ were positively associated with posttraumatic meningitis, whereas evacuation of hematoma was negatively associated with it. This study revealing the association of posttraumatic meningitis with various factors provides important clues for the prevention of posttraumatic meningitis in patients with traumatic brain injury patients with head AIS score 3 or more.

First, burr hole surgery in the emergency department was associated with posttraumatic meningitis in this study. When traumatic brain injury patients with acute epidural hematoma and/or acute subdural hematoma suffer from a rapid loss of consciousness, burr hole surgery is performed in the emergency department as an emergency procedure before damage to the brainstem becomes irreversible [23-25]. In addition, burr hole surgery is performed in the emergency department by surgeons and/or emergency physicians as an emergency procedure before patient transport from an area without neurosurgeons to a distant medical institution $[10,26]$. However, the present study did not sufficiently reveal why burr hole surgery in the emergency department was associated with the occurrence of posttraumatic meningitis.

Second, decompressive craniectomy and external ventricular drainage were also associated with posttraumatic meningitis as procedures performed during the first neurosurgical operation. It was previously reported that decompressive craniectomy reduces intracranial pressure (ICP) in patients with high ICP due to traumatic brain injury [11-15, 27, 28]. However, dura and skin can sometimes not be adequately sutured due to excessive cerebral edema in patients, whose ICP is extremely high. There were also several reports on the positive association between external ventricular drainage and meningitis [8, 9, 29]. The endocranial space, which normally has no communication with the outside, is connected with the outside by decompressive craniectomy and external ventricular drainage, and bacteria might invade the endocranial space and cause meningitis. Early cranioplasty or removal of the ventricular drain could help to prevent posttraumatic meningitis in these patients [29].

Third, repeat surgery within $48 \mathrm{~h}$ of the first surgery have previously been associated with posttraumatic meningitis. Repeat surgery is often performed in head trauma patients because of intracranial hematoma on the opposite side after 
the initial surgery or cerebral edema after brain contusion. Long operation time and repeat surgery were associated with postoperative meningitis in patients undergoing neurosurgery [9]. In addition, not only trauma but also therapeutic interventions such as surgery affect the immune response of the trauma patients [30]. Therefore, the second blow caused by the neurosurgical operation may result in cerebral edema and hematoma on the opposite side after the initial surgery. Furthermore, this second blow influencing the patient's immune response might also be associated with posttraumatic meningitis. If ICP is controlled by intensive care such as osmotic diuresis and hypothermia, decompressive craniectomy, or repeat surgery for TBI are unnecessary to control ICP. Therefore, intensive care for ICP control excluding external ventricular drainage may be helpful to prevent posttraumatic meningitis.

Finally, multiple trauma was associated with posttraumatic meningitis. Compared with single head trauma, multiple trauma is more invasive to the body and might be related to the occurrence of posttraumatic meningitis due to deterioration of the immune system in multiple trauma patients [31-34]. However, subgroup analysis showed that the factors associated with posttraumatic meningitis were different between single head injury and multiple trauma. Although the reason for this result was unclear, more invasive procedures were performed on multiple trauma patients than on single head injury patients, which could result in decreased immunity and the subsequent occurrence of posttraumatic meningitis. It is unclear why the incidence of posttraumatic meningitis was significantly lower in the trauma patients with the evacuation of hematoma, and further studies are needed. In addition, if an injury site such as that on the extremities is at risk for infection, the prevention of infection such as with the administration of antibiotics would be necessary.

\section{Limitations}

There are some limitations in this study. First, because we extracted data on meningitis from a trauma registry, we did not obtain information on how the diagnosis of meningitis was made. However, because the morbidity rate of the patients with posttraumatic meningitis was 0.4 to $1.4 \%$ in the previous studies on posttraumatic meningitis $[4,35]$, and the rate in the present study was equivalent to the rates in these studies, we believe that the diagnostic accuracy would be appropriate. Second, information on the administration of antibiotics in each patient was unknown in this study, but it would be important, as the administration of antibiotics before the occurrence of posttraumatic meningitis might lead to its prevention. However, antibiotic treatments based on the Surviving Sepsis Campaign Guidelines were performed in many intensive care units in Japan [36]. Therefore, similar administration of antibiotic treatments would have been performed on the present study patients. Third, CSF leakage in patients with basilar skull fracture was assessed in this study, but CSF leakage due to frontal sinus injury could not be assessed, because there were no data on this condition. Finally, this study was an observational study, and there may be some unknown confounding factors.

\section{Conclusions}

By use of a nationwide trauma registry in Japan, this study revealed that factors such as male sex, multiple trauma, basilar skull fracture, CSF leakage, burr hole surgery in the emergency department, burr hole surgery and craniotomy as initial operative methods, decompressive craniectomy, and external ventricular drainage, as procedures performed during the neurosurgical operation, and repeat surgery within $48 \mathrm{~h}$ of the first surgery were associated with posttraumatic meningitis in traumatic brain injury patients with head AIS score 3 or more. Our findings suggest that the prevention of infection such as the administration of antibiotics might be of help for traumatic head injury patients who require decompressive craniectomy or reoperation to prevent posttraumatic meningitis.

Acknowledgements The authors thank the emergency medical service personnel, nurses, neurosurgeon and emergency physicians who participated in the JTDB. This article was supported by the Clinical Investigator's Research Project in the Osaka University Graduate School of Medicine.

Author contributions YK analyzed the data and wrote the first draft of this manuscript. JS and KK did data-cleaning and supported analysis of the data. TK reviewed all statistical analyses and critically revised this manuscript. TH, TK, TM, JT, SN, YU, and YN interpreted the data and critically revised this manuscript. All of the authors read and approved the final manuscript.

Funding This study was supported by The General Insurance Association of Japan.

Data availability The data that support the findings of this study are available from the JTDB, but the availability of these data is restricted.

\section{Compliance with ethical standards}

Conflict of interest The authors declare that they have no competing of interests.

Ethics approval and consent to participate This study was approved by the ethics committees of the Osaka Graduate School of Medicine (No. 16260), and the requirement to obtain patients' consent to participate was waived, because the data were anonymous.

Open Access This article is distributed under the terms of the Creative Commons Attribution 4.0 International License (http://creat ivecommons.org/licenses/by/4.0/), which permits unrestricted use, 
distribution, and reproduction in any medium, provided you give appropriate credit to the original author(s) and the source, provide a link to the Creative Commons license, and indicate if changes were made.

\section{References}

1. Japan Trauma Care and Research. Japan trauma data bank annual report 2013-2017; 2018. https://www.jtcr-jatec.org/traumabank/ dataroom/data/JTDB2018e.pdf. Accessed 09 Apr 2019

2. Abu Hamdeh S, Marklund N, Lannsjö M, Howells T, Raininko R, Wikström J, et al. Extended anatomical grading in diffuse axonal injury using mri: hemorrhagic lesions in the substantia nigra and mesencephalic tegmentum indicate poor long-term outcome. J Neurotrauma. 2017;34(2):341-52.

3. Buckwold FJ, Hand R, Hansenbout RR. Hospital-acquired bacterial meningitis in neurosurgical patients. J Neurosurg. 1977;46:494-9.

4. Lau YL, Kenna AP. Post-traumatic meningitis in children. Injury. 1986;17:407-9.

5. McCracken GH, Mize S, Threlkeld N. Intraventricular gentamicin therapy in Gram-negative bacillary meningitis of infancy. Lancet. 1980;1:787-91.

6. Applebaum E. Meningitis following trauma to head and face. JAMA. 1960;173:1818-22.

7. Sonig A, Thakur JD, Chittiboina P, Khan IS, Nanda A. Is posttraumatic cerebrospinal fluid fistula a predictor of posttraumatic meningitis? A US Nationwide Inpatient Sample database study. Neurosurg Focus. 2012;32(6):E4.

8. Kourbeti IS, Jacobs AV, Koslow M, Karabetsos D, Holzman RS. Risk factors associated with postcraniotomy meningitis. Neurosurgery. 2007;60(2):317-25 (discussion 25-6).

9. Chen CH, Chang CY, Lin LJ, Chen WL, Chang YJ, Wang SH, et al. Risk factors associated with postcraniotomy meningitis: a retrospective study. Medicine. 2016;95(31):e4329.

10. Wilson MH, Wise D, Davies G, Lockey D. Emergency burr holes: "How to do it". Scand J Trauma Resusc Emerg Med. 2012;20:24.

11. Hutchinson PJ, Kolias AG, Timofeev IS, Corteen EA, Czosnyka M, Timothy $\mathrm{J}$, et al. Trial of decompressive craniectomy for traumatic intracranial hypertension. N Engl J Med. 2016;375(12):1119-30.

12. Taylor A, Butt W, Rosenfeld J, Shann F, Ditchfield M, Lewis E, et al. A randomized trial of very early decompressive craniectomy in children with traumatic brain injury and sustained intracranial hypertension. Childs Nerv Syst. 2001;17(3):154-62.

13. Albanese J, Leone M, Alliez JR, Kaya JM, Antonini F, Alliez $\mathrm{B}$, et al. Decompressive craniectomy for severe traumatic brain injury: evaluation of the effects at one year. Crit Care Med. 2003;31(10):2535-8.

14. Aarabi B, Hesdorffer DC, Ahn ES, Aresco C, Scalea TM, Eisenberg HM. Outcome following decompressive craniectomy for malignant swelling due to severe head injury. J Neurosurg. 2006;104(4):469-79.

15. Howard JL, Cipolle MD, Anderson M, Sabella V, Shollenberger D, Li PM, et al. Outcome after decompressive craniectomy for the treatment of severe traumatic brain injury. J Trauma. 2008;65(2):380-5 (discussion 5-6).

16. Butcher N, Balogh ZJ. AIS\%3e2 in at least two body regions: a potential new anatomical definition of polytrauma. Injury. 2012;43:196-9.

17. Yumoto T, Mitsuhashi T, Yamakawa Y, Iida A, Nosaka N, Tsukahara K, et al. Impact of Cushing's sign in the prehospital setting on predicting the need for immediate neurosurgical intervention in trauma patients: a nationwide retrospective observational study. Scand J Trauma Resusc Emerg Med. 2016;24(1):147.
18. Shoko T, Shiraishi A, Kaji M, Otomo Y. Effect of pre-existing medical conditions on in-hospital mortality: analysis of 20,257 trauma patients in Japan. J Am Coll Surg. 2010;211(3):338-46.

19. Nakada TA, Nakao S, Mizushima Y, Matsuoka T. Association between male sex and increased mortality after falls. Acad Emerg Med. 2015;22(6):708-13.

20. van Veen KE, Brouwer MC, van der Ende A, van de Beek D. Bacterial meningitis in diabetes patients: a population-based prospective study. Sci Rep. 2016;6:36996.

21. Danese MD, Griffiths RI, Dylan M, Yu HT, Dubois R, Nissenson AR. Mortality differences among organisms causing septicemia in hemodialysis patients. Hemodial Int. 2006;10(1):56-62.

22. Matsuyama T, Kitamura T, Katayama Y, Hirose T, Kiguchi T, Sado J, et al. Motor vehicle accident mortality by elderly drivers in the super-aging era: a nationwide hospital-based registry in Japan. Medicine. 2018;97(38):e12350.

23. Motohashi O, Kameyama M, Shimosegawa Y, Fujimori K, Sugai $\mathrm{K}$, Onuma T. Single burr hole evacuation for traumatic acute subdural hematoma of the posterior fossa in the emergency room. $\mathrm{J}$ Neurotrauma. 2002;19(8):993-8.

24. Smith SW, Clark M, Nelson J, Heegaard W, Lufkin KC, Ruiz E. Emergency department skull trephination for epidural hematoma in patients who are awake but deteriorate rapidly. J Emerg Med. 2010;39(3):377-83

25. Ren H, Yin L, Ma L, Wei M, Ma X. Emergency bedside evacuation of a subset of large postoperative epidural hematomas after neurosurgical procedures. Medicine. 2018;97(30):e11475.

26. Luck T, Treacy PJ, Mathieson M, Sandilands J, Weidlich S, Read D. Emergency neurosurgery in Darwin: still the generalist surgeons' responsibility. ANZ J Surg. 2015;85(9):610-4.

27. Timofeev I, Czosnyka M, Nortje J, Smielewski P, Kirkpatrick P, Gupta A, et al. Effect of decompressive craniectomy on intracranial pressure and cerebrospinal compensation following traumatic brain injury. J Neurosurg. 2008;108(1):66-73.

28. Cooper DJ, Rosenfeld JV, Murray L, Arabi YM, Davies AR, D'Urso P, et al. Decompressive craniectomy in diffuse traumatic brain injury. N Engl J Med. 2011;364(16):1493-502.

29. Murthy SB, Moradiya Y, Shah J, Hanley DF, Ziai WC. Incidence, predictors, and outcomes of ventriculostomy-associated infections in spontaneous intracerebral hemorrhage. Neurocrit Care. 2016;24(3):389-96.

30. Peitzman AB, Yealy DM, Fabian TC, Rhodes M, Schwab CW. The trauma manual: trauma and acute care surgery. 4 th ed. Philadelphia: Lippincott Williams and Wilkins; 2012. p. 14-15.

31. Moore FA, Moore EE. Evolving concepts in the pathogenesis of postinjury multiple organ failure. Surg Clin N Am. 1995;75:257-77.

32. Paterson HM, Murphy TJ, Purcell EJ, et al. Injury primes the innate immune system for enhanced Toll-like receptor reactivity. J Immunol. 2003;181:1473-83.

33. Fan J, Marshall JC, Jumenez M, et al. Hemorrhagic shock primes for increased expression of cytokine-induced neutrophil chemoattractant in the lung: role in pulmonary inflammation following lipopolysaccharide. J Immunol. 1998;161:440-7.

34. Papia G, Fan J, Kapus A, et al. Altered inhibitory kappaBalpha expression in LPS-stimulated alveolar macrophages following resuscitated hemorrhagic shock. SHOCK. 2011;35:171-7.

35. Baltas I, Tsoulfa S, Sakellariou R, Vogas V, Fylaktakis M, Kondodimou A. Posttraumatic meningitis: bacteriology, hydrocephalus, and outcome. Neurosurgery. 1994;35(3):422-6.

36. Abe T, Ogura H, Shiraishi A, Kushimoto S, Saitoh D, Fujishima $\mathrm{S}$, et al. Characteristics, management, and in-hospital mortality among patients with severe sepsis in intensive care units in Japan: the FORECAST study. Crit Care. 2018;22(1):322. 